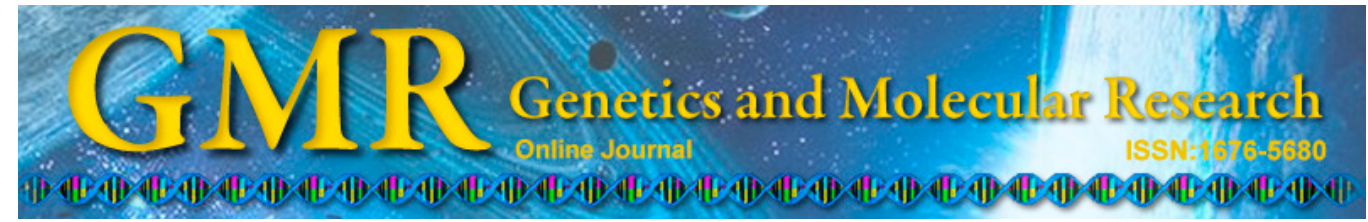

\title{
Analysis of the expression of HMGB-1, CXCL16, miRNA-30a, and TGF- $\beta 1$ in primary nephritic syndrome patients and its significance
}

\author{
W.J. Wang ${ }^{1 *}$, X.Q. Qu ${ }^{2 *}$, X.M. Yu ${ }^{3}$, W. Lv ${ }^{1}$ and H.Y. Yu ${ }^{4}$ \\ ${ }^{1}$ Nephrology Department of PLA, 89th Hospital, Weifang, China \\ ${ }^{2}$ Orthopaedic Institute of PLA, 89th Hospital, Weifang, China \\ ${ }^{3}$ Department of Clinical Laboratory, The People's Hospital of Gaomi City, \\ Gaomi, Shandong, China \\ ${ }^{4}$ Department of Urology of PLA, 89th Hospital, Weifang, China \\ *These authors contributed equally to this study. \\ Corresponding author: H.Y. Yu \\ E-mail: HaiyiYu20009@126.com
}

Genet. Mol. Res. 14 (3): 9841-9848 (2015)

Received December 17, 2014

Accepted May 29, 2015

Published August 19, 2015

DOI http://dx.doi.org/10.4238/2015.August.19.17

\begin{abstract}
We investigated the expression levels of high-mobility group box protein 1 (HMGB-1), CXC chemokine ligand 16 (CXCL16), microRNA (miRNA)-30a and transforming growth factor $\beta 1$ (TGF- $\beta 1$ ) in primary nephritic syndrome (PNS) patients and the clinical significance of this expression. A total of 56 patients with PNS were included in the PNS group, while 50 healthy subjects formed the normal control group. Serum levels of HMGB-1, CXCL16, miRNA-30a, and urinary TGF- $\beta 1$ concentrations were quantified along with other biochemical indices, including serum albumin, triglyceride (TG), total cholesterol (TC), lowdensity lipoprotein (LDL), high-density lipoprotein, and urinary proteins. The correlation between levels of HMGB-1, CXCL16, miRNA-30a, and TGF- $\beta 1$ and biochemical indexes was further analyzed. PNS group
\end{abstract}


patients had significantly higher levels of HMGB-1, CXCL16, miRNA$30 \mathrm{a}$, and TGF- $\beta 1$ compared to the control group $(\mathrm{P}<0.05)$. PNS patients also had higher 24-h urinary protein, TG, TC, and LDL levels but lower serum albumin compared to subjects in the control group $(\mathrm{P}<0.05)$. Serum HMGB-1, CXCL16, miRNA-30a, and urinary TGF- $\beta 1$ levels were all negatively correlated with serum albumin levels, but were positively correlated with TG, TC, LDL, and 24-h urinary protein (P $<0.05$ in all cases). Additionally, a positive correlation existed among serum HMGB-1, CXCL16, miRNA-30a, and urinary TGF- $\beta 1$ levels (P $<0.01)$. HMGB-1, CXCL16, miRNA-30a, and urinary TGF- $\beta 1$ were highly expressed in PNS patients and may play important roles in the pathogenesis and development of PNS.

Key words: High-mobility group box protein 1; MicroRNA-30a; CXC chemokine ligand 16; Primary nephritic syndrome;

Transforming growth factor- $\beta 1$

\section{INTRODUCTION}

Primary nephritic syndrome (PNS) is a class of systematic inflammation-related immune disorders caused by multiple factors, with a pathological basis consisting of elevated permeability of the glomerular basement membrane. Currently, little has been known about the pathogenesis of PNS, although cellular mechanisms, inflammatory mediators, immune disorders, and genetic molecules are involved, with major contributions from immune induction and inflammatory damage (Gigante et al., 2014; Wu et al., 2014). As a result, the role of immune inflammatory response-mediating factors, including nuclear factor- $\mathrm{\kappa B}$, tumor necrosis factor- $\alpha$, interleukin-1, interleukin- 6 , and interleukin- 8 , in the pathogenesis and development of PNS has become an active field in clinical studies and has received considerable attention (Urbonaviciute et al., 2008).

In recent years, novel cytokines and biomarkers have been developed as more comprehensive studies have examined PNS pathogenesis, and significant progress has been made in molecular biology, thereby providing new pathways for the study of PNS pathogenesis (Urbonaviciute et al., 2007). High-mobility group box protein 1 (HMGB-1) was found to be a novel inflammatory cytokine and has been reported to be highly expressed in the serum of chronic renal dysfunction patients (Li et al., 2011). CXC chemokine ligand 16 (CXCL16) is the only known chemokine that can exert its chemotactic activity in both transmembrane and soluble forms. CXCL16 has been found to be closely related to acute renal tubular injury (Stojković et al., 2014). The relationship between CXCL16 and PNS occurrence and its developmental mechanism has not been thoroughly examined. MicroRNA (miRNA)-30a is a biomarker that widely participates in various physiological processes, including cell proliferation, apoptosis, immune response, and metabolism. Previous studies have confirmed the close relationship between miRNA-30a and PNS pathogenesis. Transforming growth factor (TGF)- $\beta 1$ is a pluripotent cytokine with important roles in the progression of renal disease (Zhen and Cao, 2014), but few studies have examined its potential roles in the pathogenesis and progression of PNS. Based on these previous studies, we measured the expression levels 
of HMGB-1, CXCL16, miRNA-30a, and TGF- $\beta 1$ to provide information useful for the development of novel drug targets against PNS.

\section{MATERIAL AND METHODS}

\section{Patient information}

A total of 56 PNS patients admitted to our hospital between June 2013 and June 2014 were included in the PNS group. All included patients fit the diagnostic standard stipulated by the symposium of typing, diagnostic, and treatment standards of primary glomerular disease in 1992 (Aloni et al., 2014) and had no history of PNS. There were 32 males and 24 females, aged 32-75 years (average $=43.5 \pm 5.4$ years). Another cohort of 50 healthy adults who received routine physical examinations in our hospital was included as the control group. There were 28 males and 22 females in the control group, aged 34-76 years (average $=44.2 \pm 5.6$ years). There was no statistically significant difference regarding age and gender between the PNS group and the control group patients $(\mathrm{P}>0.05)$. The following exclusion criteria were used: 1) patients complicated with other secondary nephritic syndromes; 2) patients with tumors, severe kidney, liver or heart disease, or with inflammatory disease within last 3 months; 3 ) had received immunosuppressant or glucocorticoid medicines within 1 week.

\section{Sample collection}

First, $5 \mathrm{~mL}$ fasting peripheral venous blood was collected from PNS patients in the morning 2 days after being admitted and from control subjects on the same day of physical examinations. Blood samples stored in ethylenediaminetetraacetic acid anti-coagulant tubes were centrifuged to collect the serum, which was stored at $-70^{\circ} \mathrm{C}$. Additionally, $5 \mathrm{~mL} 24-\mathrm{h}$ urine samples (from 8:00 am on the same day of blood sample collection to 8:00 am on the next day) from both groups were collected.

\section{Biochemical assays}

For biochemical assays, $3 \mathrm{~mL}$ blood samples were quantified for biochemical indices, including serum albumin, triglyceride (TG), total cholesterol (TC), low-density lipoprotein (LDL), and high-density lipoprotein using a Beckman Coulter automatic biochemical analyzer (Model AU5800, Brea, CA, USA). Additionally, $3 \mathrm{~mL}$ urine samples were quantified for 24-h urinary proteins using the chemical method. Furthermore, $2 \mathrm{~mL}$ blood samples were quantified for serum HMGB-1 and CXCL16 levels using an enzyme-linked immunosorbent assay following the kit manual instructions. Briefly, standard samples were diluted and test samples were added. After warm incubation and washing, enzyme reagents were added for further incubation. Chromogenic substrates were added for color development, which was quantified after the stopping point. Total RNA was extracted from the blood samples and measured for the expression level of miRNA-30a using real-time fluorescent quantitative polymerase chain reaction. Briefly, miRNA-30a-specific primers (forward: 5'-AAATG GTGAA GGTCG GTGTG-3'; reverse: 5'-TGAAG GGGTC GTTGA TGG-3') were used for in vitro reverse transcription reactions using $100 \mathrm{mM}$ dNTP as the substrate. miRNA concentrations were detected using a real-time fluorescent method with the amplification curve of miRNA-30a as shown in 
Figure 1. All polymerase chain reaction data were normalized and calculated for the relative expression level of miRNA-30a using $2^{-\Delta \Delta C T}$ method $\left(\Delta \mathrm{CT}=\mathrm{CT}_{\text {miR-30a }}-\mathrm{CT}_{\text {cel-miR-39 }}\right)$. Finally, urinary TGF- $\beta 1$ levels were assayed by enzyme-linked immunosorbent assay kit from $2 \mathrm{~mL}$ urine samples following the manual instruction (Wang et al., 2014).

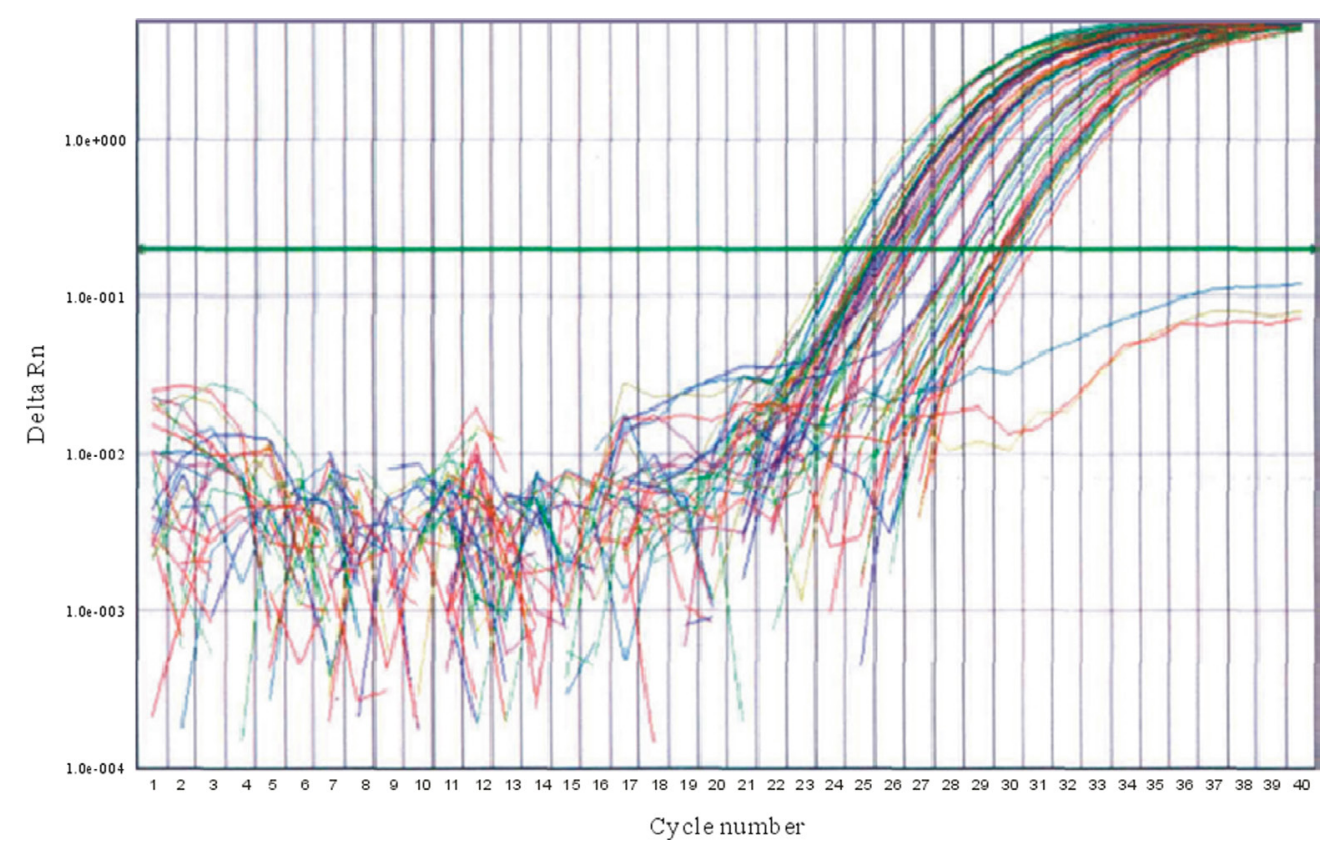

Figure 1. Fluorescent quantitative PCR amplification curves of miRNA-30a.

\section{Statistical analysis}

The SPSS 20.0 software was used to process all collected data (SPSS, Inc., Chicago, IL, USA), in which quantitative data are reported as means \pm SE. The Student $t$-test was used to compare all biochemical indices, including HMGB-1, CXCL16, miRNA-30a, and TGF- $\beta 1$ between the PNS and control groups. Spearman correlation analysis was further employed to analyze the relationship between the levels of HMGB-1, CXCL16, miRNA$30 \mathrm{a}$, and TGF- $\beta 1$ and all biochemical indices. Statistical significance was defined when $\mathrm{P}$ $<0.05$.

\section{RESULTS}

\section{Comparisons of serum HMGB-1, CXCL16, miRNA-30a, and urinary TGF- $\beta 1$ levels}

As shown in Table 1, PNS patients had significantly higher serum HMGB-1, CXCL16, miRNA-30a, and urinary TGF- $\beta 1$ levels compared to subjects in the normal group $(\mathrm{P}<0.05)$. 
Table 1. Serum HMGB-1, CXCL16, miRNA-30a, and urinary TGF- $\beta 1$ levels.

\begin{tabular}{lcc}
\hline Parameter & PNS group $(\mathrm{N}=56)$ & Control group $(\mathrm{N}=50)$ \\
\hline HMGB-1 $(\mathrm{ng} / \mathrm{L})$ & $28.93 \pm 6.94^{*}$ & $10.22 \pm 2.17$ \\
CXCL16 $(\mathrm{ng} / \mathrm{L})$ & $0.42 \pm 0.07^{*}$ & $0.31 \pm 0.04$ \\
miRNA-30a $(\Delta \mathrm{CT})$ & $2.35 \pm 0.42^{*}$ & $1.02 \pm 0.04$ \\
TGF- $\beta 1(\mu \mathrm{g} / \mathrm{L})$ & $271.63 \pm 102.34^{*}$ & $49.36 \pm 28.35$ \\
\hline
\end{tabular}

PNS = primary nephritic syndrome. Data are reported as means \pm SE. $* \mathrm{P}<0.05$ compared to control group.

\section{Biochemical indices between groups}

In PNS patients, 24-h urinary proteins, TG, TC, and LDL levels were all significantly higher than those in the control group $(\mathrm{P}<0.05$; Table 2$)$. The serum albumin level, however, was lower in PNS patients compared to normal subjects $(\mathrm{P}<0.05)$. No statistically significant difference was found in high-density lipoprotein levels between the 2 groups $(\mathrm{P}>0.05)$.

Table 2. Biochemical indices between primary nephritic syndrome (PNS) patients and normal controls.

\begin{tabular}{lcr}
\hline Index & PNS group $(\mathrm{N}=56)$ & Control group $(\mathrm{N}=50)$ \\
\hline Serum albumin $(\mathrm{g} / \mathrm{L})$ & $20.61 \pm 4.04^{*}$ & $38.55 \pm 2.91$ \\
TG $(\mathrm{mM})$ & $3.29 \pm 1.52^{*}$ & $1.05 \pm 0.32$ \\
TC $(\mathrm{mM})$ & $9.15 \pm 2.05^{*}$ & $3.82 \pm 1.33$ \\
LDL $(\mathrm{mM})$ & $5.67 \pm 2.41^{*}$ & $2.63 \pm 1.66$ \\
HDL $(\mathrm{mM})$ & $1.62 \pm 0.82$ & $1.68 \pm 0.84$ \\
24-h urinary protein $(\mathrm{g} / \mathrm{L})$ & $6.74 \pm 3.15^{*}$ & - \\
\hline
\end{tabular}

Data are reported as means $\pm \mathrm{SE} . * \mathrm{P}<0.05$ compared to control group.

\section{Correlation analysis between levels of HMGB-1, CXCL16, miRNA-30a, and TGF- $\beta 1$ and biochemical indices}

Table 3 shows that the levels of HMGB-1, CXCL161, miRNA-30a, and TGF- $\beta 1$ were negatively correlated with serum albumin levels $(\mathrm{r}=-5.432,-5.246,-3.142$, and $-2.813 ; \mathrm{P}<$ 0.01 in all cases) and were positively related to TG $(\mathrm{r}=0.347,0.365,0.293$, and $0.271 ; \mathrm{P}<$ 0.05 in all cases), TC $(\mathrm{r}=0.516,0.323,0.318$, and $0.353 ; \mathrm{P}<0.01$ in all cases $), \mathrm{LDL}(\mathrm{r}=0.258$, $0.273,0.219$, and $0.213 ; \mathrm{P}<0.05$ in all cases $)$, and 24 -h urinary proteins $(\mathrm{r}=0.764,0.736$, 0.675 , and $0.643 ; \mathrm{P}<0.01$ in all cases). Spearman correlation analysis also revealed positive relationships among HMGB-1, CXCL16, miRNA-30a, and TGF- $\beta 1$ levels $(\mathrm{r}=0.643, \mathrm{P}<0.01)$.

\begin{tabular}{|c|c|c|c|c|c|c|c|c|}
\hline \multirow[t]{2}{*}{ Biochemical index } & \multicolumn{2}{|c|}{ HMGB-1 } & \multicolumn{2}{|c|}{ CXCL16 } & \multicolumn{2}{|c|}{ miRNA-30a } & \multicolumn{2}{|c|}{ TGF- $\beta 1$} \\
\hline & $\mathrm{r}$ value & $\mathrm{P}$ value & $\mathrm{r}$ value & $\mathrm{P}$ value & r value & P value & $r$ value & $\mathrm{P}$ value \\
\hline Serum albumin & -5.342 & 0.000 & -5.246 & 0.000 & -3.142 & 0.006 & -2.813 & 0.008 \\
\hline TG & 0.347 & 0.005 & 0.365 & 0.004 & 0.293 & 0.018 & 0.271 & 0.026 \\
\hline $\mathrm{TC}$ & 0.516 & 0.000 & 0.323 & 0.009 & 0.318 & 0.007 & 0.353 & 0.005 \\
\hline LDL & 0.258 & 0.028 & 0.273 & 0.025 & 0.219 & 0.029 & 0.213 & 0.032 \\
\hline HDL & -0.047 & 0.781 & -0.042 & 0.794 & -0.044 & 0.913 & -0.054 & 0.813 \\
\hline Urinary protein & 0.764 & 0.000 & 0.736 & 0.000 & 0.675 & 0.000 & 0.643 & 0.000 \\
\hline
\end{tabular}




\section{DISCUSSION}

As an abundantly expressed and highly conserved protein, HMGB-1 is both actively secreted and passively released (Wu et al., 2010). During passive release, HMGB-1 exerts negative effects on the body such as the induction of a systematic inflammatory response, destruction of epithelial barriers, and impairments in organ functions. Thus, HMGB-1 has been referred to as a "risk signal" based on its inflammatory tissue damages and cell necrosis, and may participate in cell necrosis as an important inflammatory cytokine (Robinson et al., 2013). This study revealed higher serum expression level of HMGB-1 in PNS patients than in control subjects (Table 1), suggesting that HMGB-1 is an important factor in the pathogenesis and progression of PNS. Although the detailed role of HMGB-1 in PNS pathogenesis has not been elucidated, its expression in the serum of renal disease patients and potential mechanisms have been widely reported. For example, the Toll-like receptor 4 signaling pathway is involved in the HMGB-1-activated peripheral blood mononuclear cell pathway, which further leads to the release of inflammatory cytokines, causing the failure of multiple organs, particularly the kidneys (Gabryel et al., 2011). Additionally, we found that HMGB-1 was negatively correlated with serum albumin levels and was positively related with TG, TC, LDL, and 24-h urinary proteins (Table 3), suggesting a positive relationship between HMGB-1 expression and renal dysfunction. This is consistent with the results of previous studies, which reported that acute renal injuries significantly elevated HMGB-1 levels, but these levels can be improved by anti-inflammation treatment with glutamine (Feng et al., 2008). Thus, the HMGB-1 induced inflammatory response may be an important mechanism underlying the pathogenesis and progression of PNS.

CXCL16 is the only known chemokine that exists as both a transmembrane and a soluble molecule. We found higher serum CXCL16 levels in PNS patients than in healthy subjects (Table 1), suggesting potential roles for HMGB-1 in the pathogenesis and progression of PNS. Numerous studies have examined the role of CXCL16 in various acute and chronic renal diseases. The blocking of CXCL16, for example, can significantly inhibit the infiltration of monocytes and macrophages during the advanced stage or acute inflammation stage of nephritis, thereby lowering urinary protein levels and ameliorating glomerular injuries (Han et al., 2014). The current study revealed a negative relationship between CXCL16 levels and serum albumin, as well as positive relationships between CXCL16 levels and indices such as TG, TC, LDL, and 24-h urinary proteins (Table 3), suggesting a positive relationship between CXCL16 levels and the degree of renal injuries. Consistent with such observations, when the body is under inflammatory response, the CXCL16-induced specific chemotactic response can help highly expressed T lymphocytes to migrate to the inflammatory site, further advancing the inflammatory response (Kalliomaki et al., 2014). Another study in glomerulonephritis model rats found high expression levels of CXCL16 in glomerular endothelial cells, where CXCL16 exerted a critical role in glomerular infiltration (Lee et al., 2014). Therefore, CXCL16 may induce inflammatory cells to migrate to the inflammatory renal tissues via its inherent chemotactic activity, further advancing the condition of PNS (Wittel et al., 2014).

As a family of single-stranded non-coding small RNA molecules consisting of 18-24 nucleotides, miRNA-30a is widely distributed and has a highly conserved sequence. Clinical studies have revealed an inhibitory role of miRNA-30a in the transformation of epithelial cells into mesenchymal cells. miRNA-30a showed decreased expression in non-small cell lung cancer patients. However, the exact origin, expressional profiles, and pathological roles of miRNA- 
30a in PNS patients remain unknown. This study revealed higher concentrations of miRNA30a in the serum of PNS patients compared to controls (Table 1), in addition to a negative relationship with serum albumin levels and positive correlations with TG, TC, LDL, and 24-h urinary proteins (Table 3). These results suggest a potential role for miRNA-30a in the pathogenesis and development of PNS, providing valuable guidance for diagnosis and treatment.

As suggested by previous studies, the high expression of TGF- $\beta 1$ constitutes the pathological basis of tissue fibrosis and plays an important role in the progression of renal diseases (Liu et al., 2014). Renal cells are the major origin of urinary TGF- $\beta 1$, as well as its functional targets (Chun et al., 2014). We observed higher urinary TGF- $\beta 1$ levels in PNS patients than in control subjects (Table 1), as well as a negative relationship between serum albumin level and positive relationships with TG, TC, LDL, and 24-h urinary proteins (Table 3). These data collectively indicate a potential role of TGF- $\beta 1$ in PNS occurrence and development (Papadimitriou et al., 2014). Therefore, it is critical to closely monitor urinary TGF- $\beta 1$ levels in order to prevent kidney fibrosis in PNS patients (Dursun et al., 2013). Additionally, we found a positive relationship among HMGB-1, CXCL16, and urinary TGF- $\beta 1$ levels, suggesting a potential synergistic effect of these factors in the pathogenesis and progression of PNS (Ladapo et al., 2014), although the detailed mechanisms remain unknown.

In summary, HMGB-1, CXCL16, and TGF- $\beta 1$ are highly expressed in PNS patients, suggesting their potencies in the disease course of PNS. These factors, as well as miRNA-30a, may be used as clinical indices for monitoring treatment efficacy and predicting prognosis, and as targets for the development of next-generation drugs against PNS, although more comprehensive studies are required to elucidate their potential synergistic effects and detailed mechanisms.

\section{REFERENCES}

Aloni MN, Sysleyne LM, Ekulu PM, Babio FL, et al. (2014). The challenges of caring for children with nephrotic syndrome in a tertiary institution in the Democratic Republic of Congo. Acta Paediatr. 103: e365-e369.

Chun JN, Kim SY, Park EJ, Kwon EJ, et al. (2014). Schisandrin B suppresses TGF $\beta 1$-induced stress fiber formation by inhibiting myosin light chain phosphorylation. J. Ethnopharmacol. 152: 364-371.

Dursun H, Noyan A, Matyar S, Buyukcelik M, et al. (2013). Association of eNOS gene intron $4 \mathrm{a} / \mathrm{b}$ VNTR polymorphisms in children with nephrotic syndrome. Gene 522: 192-195.

Feng Y, Yang Q, Xu J, Qian G, et al. (2008). Effects of HMGB1 on PMN apoptosis during LPS-induced acute lung injury. Exp. Mol. Pathol. 85: 214-222.

Gabryel B, Bielecka A, Bernacki J, Labuzek K, et al. (2011). Immunosuppressant cytoprotection correlates with HMGB1 suppression in primary astrocyte cultures exposed to combined oxygen-glucose deprivation. Pharmacol. Rep. 63: $392-402$.

Gigante A, Barbano B, Sardo L, Martina P, et al. (2014). Hypercoagulability and nephrotic syndrome. Curr. Vasc. Pharmacol. 12: 512-517.

Han EC, Lee J, Ryu SW and Choi C (2014). Tumor-conditioned Gr-1(+)CD11b(+) myeloid cells induce angiogenesis through the synergistic action of CCL2 and CXCL16 in vitro. Biochem. Biophys. Res. Commun. 443: 1218-1225.

Kalliomaki M, Rajala S, Elamo H, Ashorn M, et al. (2014). Increased expression of CXCL16, a bacterial scavenger receptor, in the colon of children with ulcerative colitis. J. Crohns Colitis 8: 1222-1226.

Ladapo TA, Esezobor CI and Lesi FE (2014). High steroid sensitivity among children with nephrotic syndrome in southwestern Nigeria. Int. J. Nephrol. 2014: 350640.

Lee HS, Hong JE, Kim EJ and Kim SH (2014). Escin suppresses migration and invasion involving the alteration of CXCL16/CXCR6 axis in human gastric adenocarcinoma AGS cells. Nutr. Cancer 66: 938-945.

Li J, Gong Q, Zhong S, Wang L, et al. (2011). Neutralization of the extracellular HMGB1 released by ischaemic damaged renal cells protects against renal ischaemia-reperfusion injury. Nephrol. Dial. Transplant. 26: 469-478.

Liu SD, Shen QM and Lv CF (2014). Reversible posterior leukoencephalopathy syndrome in children with nephrotic syndrome: a case report. Chin. Med. Sci. J. 29: 55-57. 
Papadimitriou A, Peixoto EB, Silva KC, et al. (2014). Increase in AMPK brought about by cocoa is renoprotective in experimental diabetes mellitus by reducing NOX4/TGFß-1 signaling. J. Nutr. Biochem. 25: 773-784.

Robinson AP, Caldis MW, Harp CT, Goings GE, et al. (2013). High-mobility group box 1 protein (HMGB1) neutralization ameliorates experimental autoimmune encephalomyelitis. J. Autoimmun. 43: 32-43.

Stojković L, Stanković A, Djurić T, Dinčić E, et al. (2014). The gender-specific association of CXCL16 A181V gene polymorphism with susceptibility to multiple sclerosis, and its effects on PBMC mRNA and plasma soluble CXCL16 levels: preliminary findings. J. Neurol. 261: 1544-1551.

Urbonaviciute V, Furnrohr BG, Weber C, Haslbeck M, et al. (2007). Factors masking HMGB1 in human serum and plasma. J. Leukoc. Biol. 81: 67-74.

Urbonaviciute V, Furnrohr BG, Meister S, Munoz L, et al. (2008). Induction of inflammatory and immune responses by HMGB1-nucleosome complexes: implications for the pathogenesis of SLE. J. Exp. Med. 205: 3007-3018.

Wang L, Sun S, Zhou A, Yao X, et al. (2014). oxLDL-induced lipid accumulation in glomerular podocytes: role of IFN- $\gamma$, CXCL16, and ADAM10. Cell Biochem. Biophys. 70: 529-538.

Wittel UA, Schmidt AI, Puolakkainen P, Seifert GJ, et al. (2014). Chemokine ligand CXCL16 is an indicator of infected necrosis in necrotizing pancreatitis. Pancreatology 14: S55.

Wu H, Ma J, Wang P, Corpuz TM, et al. (2010). HMGB1 contributes to kidney ischemia reperfusion injury. J. Am. Soc. Nephrol. 21: 1878-1890.

Wu JB, Ye SF, Liang CL, Li YC, et al. (2014). Qi-Dan Fang ameliorates adriamycin-induced nephrotic syndrome rat model by enhancing renal function and inhibiting podocyte injury. J. Ethnopharmacol. 151: 1124-1132.

Zhen G and Cao X (2014). Targeting TGF $\beta$ signaling in subchondral bone and articular cartilage homeostasis. Trends Pharmacol. Sci. 35: 227-236. 